\title{
Neuf bonnes raisons de faire signer l'initiative
}

Comité d'initiative Transparence de l'assurance-maladie

http://

initiative-transparence.ch

\section{Deux assurances aux vocations différentes}

L'assurance-maladie obligatoire (LAMal) est une assurance sociale qui doit respecter la solidarité et est financée principalement par un prélèvement obligatoire sous forme de primes par tête, assimilable à un impôt. Elle comprend un catalogue de prestations identique pour tous.

L'assurance complémentaire (LCA) est, quant à elle, facultative et permet à l'assureur de réaliser des bénéfices. Elle l'autorise également à refuser un assuré ou exclure du contrat certaines prestations en fonction de son état de santé.

Les assureurs-maladie ont actuellement le droit de pratiquer conjointement ces deux activités.

\section{Transparence économique et surveillance insuffisantes}

L'assurance de base doit être gérée de manière entièrement transparente et ne peut être liée à une autre activité économique. Cette transparence est indispensable afin de s'assurer d'une gestion saine et économique de l'argent des primes. Pourtant, la surveillance de ces primes telle qu'elle est exercée par l'OFSP est insatisfaisante et inefficace dans le système actuel. Cet office n'affecte que 15 fonctionnaires au contrôle des 81 assureurs-maladie qui encaissent les 20 milliards de francs de primes de l'assurance obligatoire (chiffres OFSP 2009).

En dépit des critiques émises depuis plusieurs années, les primes ne sont pas fixées de manière transparente et ne correspondent pas aux coûts réels. L'affaire des réserves excessives dans certains cantons et leur transfert vers d'autres à des fins de stratégie commerciale démontre que la gestion des assureursmaladie n'est pas équitable.

\section{Fausses synergies et vraie opacité}

Si l'assurance-maladie obligatoire peut être efficacement gérée par des organismes privés comme les caisses actuelles, celles-ci doivent satisfaire à une transparence complète de leur gestion. Or les bilans consolidés publiés par les assureurs-maladie ne permettent pas d'attester de la conformité de leurs pratiques comptables selon la LAMal, leurs fiduciaires n'étant en effet pas tenues de la vérifier. L'amalgame que les assureurs-maladie réalisent en exploitant avec le même personnel et dans la même infrastructure (bâtiments, moyens informatiques) des assurances

\begin{abstract}
L'initiative populaire fédérale «Pour la transparence de l'assurance-maladie»

Les médecins des trois régions linguistiques de la Suisse, soutenus par la Fédération des médecins suisses (FMH), lancent une initiative populaire fédérale qui vise à imposer une séparation complète entre l'assurance-maladie sociale obligatoire, et les assurances complémentaires. Ainsi, les caisses-maladie ne pourront plus proposer ces deux types d'assurance, qui relèvent de règles légales différentes. Elles devront donc opter pour I'une ou pour l'autre de ces activités.
\end{abstract}

complémentaires ne permet en aucun cas une véritable transparence. L'opacité générée par la cohabitation de deux régimes d'assurance aux vocations opposées se retrouve au cœur d'un système devenu dysfonctionnel. Conjuguées à une surveillance défaillante, ces «synergies» poussent non seulement les assureurs-maladie à sélectionner les risques, mais incitent fortement au transfert de charges, de prestations et de fonds vers l'assurance de base, contribuant à renchérir artificiellement les primes. L'opacité des flux financiers au sein des grandes caisses-maladie est telle qu'elle échappe désormais à tout contrôle politique. Ce manque de transparence se répercute jusque dans la structure juridique adoptée par certains grands groupes d'assurance dans le but à peine dissimulé de mieux se soustraire à la surveillance de l'OFSP.

En dépit de leurs protestations de bonne foi, les assureurs opposent un refus net à toute tentative d'y voir clair au prétexte d'un «secret des affaires» qu'ils entendent appliquer à la gestion d'un prélèvement social obligatoire.

\section{La conclusion d'une assurance complémen- taire ne doit pas être liée à l'assurance de base}

Le catalogue des soins remboursés par l'assurance de base est le même pour tous. L'assureur exerçant la complémentaire dispose par conséquent de tous les éléments nécessaires pour distinguer la part des coûts lui incombant de celle relevant de l'assurance obligatoire. Maintenir les deux contrats auprès du même assureur est donc inutile. Pour l'assuré, une assurance 
complémentaire de qualité sera celle qui s'occupe de cette ventilation.

L'assuré doit être libre de s'assurer auprès du meilleur assureur de base, sans se sentir lié par l'assurance complémentaire. Il n'est pas acceptable qu'il soit dissuadé de changer d'assurance de base pour bénéficier de primes meilleur marché, comme la loi le lui permet, parce que son assureur complémentaire le menace d'une augmentation de prime ou d'une suppression de rabais. Ce lien obligé induit donc une changer d'assurance de base. Pourtant, de nombreux témoignages notamment rapportés dans les médias démontrent que ces données, strictement protégées en théorie, sont largement disponibles au sein d'une même assurance puisque le même personnel est souvent chargé de traiter les deux régimes d'assurance. La réforme de la tarification hospitalière (DRG) qui entrera en vigueur en 2012 va accroître l'accès des assureurs aux données sensibles relatives à la santé des assurés et augmentera considérablement le risque

\section{«L'assuré doit être libre de s'assurer auprès du meilleur assureur de base, sans se sentir lié par l'assurance complémentaire.»}

distorsion importante de la concurrence aux dépens des assurés.

\section{Sélection des risques, tracasseries et tromperies}

Contrairement à l'assurance de base où l'assureur a l'obligation d'accepter tous les assurés sans condition préalable, l'assurance complémentaire peut pratiquer la sélection des risques au moyen d'un questionnaire ou un examen de santé. De multiples témoignages indiquent pourtant que les patients coûteux, pour des raisons indépendantes de leur volonté, ne reçoivent pas de réponses à leurs demandes d'affiliation pour l'assurance de base ou constatent que les lignes téléphoniques de l'assureur ne répondent plus dès lors qu'ils ont été identifiés comme «mauvais risques». Dans le but de trier les «bons risques» certains assureurs profitent d'un changement de caisse pour soutirer aux assurés des informations relatives à leur état de santé de façon illégale ou au moyen d'une petite complémentaire-alibi. D'après la plupart des experts, il est fort improbable que la nouvelle compensation des risques prévue pour 2012 mette un terme à ces dérives.

On voit donc que les «synergies» avancées par les assureurs-maladie comme principal motif en faveur du statu quo ne profitent en réalité qu'à eux-mêmes et s'exercent au détriment des assurés. Elles réduisent l'assurance de base au rang de produit d'appel pour l'assurance complémentaire. Un coût annuel de près de 100 millions de francs pour les seuls frais de publicité et d'acquisition de nouveaux assurés serait ainsi généré pour la vente d'un produit pourtant identique chez tous les assureurs.

\section{La protection des données n'est pas respectée}

Les informations auxquelles l'assureur a accès dans le domaine des complémentaires ne devraient pas permettre une mise à l'écart de la personne qui désire de voir celles-ci utilisées à leur détriment. On voit donc que cette double activité constitue en vérité une épée de Damoclès pouvant priver l'assuré d'un accès à une couverture complémentaire ou le confronter à un refus de remboursement de prestations.

\section{Les assurés ignorent les différences entre les deux branches d'assurance}

L'assurance-maladie obligatoire relève de la loi sur l'assurance-maladie (LAMal), tandis que les assurances complémentaires dépendent de la loi sur le contrat d'assurance (LCA). Lorsqu'un même contrat prévoit une couverture combinée, il est fort difficile pour le novice de savoir quelles règles s'appliquent à quoi. Là également, l'impossibilité de proposer des contrats mixtes contribuera à la transparence et empêchera que cette confusion soit mise à profit pour récolter des données personnelles de façon plus ou moins licite.

\section{L'avenir de I'assurance-maladie est en jeu}

Le mode de financement actuel de l'assurance-maladie obligatoire se traduit par des primes qui deviennent difficilement supportables pour un nombre croissant de personnes, notamment les familles, et tous les scénarios rendent vraisemblable une poursuite de l'augmentation des coûts de la santé. Afin de les contenir, des réformes dans l'offre des soins sont en cours qui devront pouvoir s'appuyer sur un système de financement sain si elles entendent pouvoir s'appliquer avec efficacité. La transparence de l'assurance-maladie est par conséquent la condition indispensable à la restauration d'un lien de confiance et d'une crédibilité, sans lesquels il sera impossible de faire progresser durablement les réformes dont notre système de santé a besoin afin de parvenir à une modération des primes.

Les débats politiques ont démontré qu'un véritable réseau de clientélisme politique a été mis en place à Berne par les assureurs afin d'accroître leur 
mainmise sur le système de santé et bloquer toute réforme allant dans le sens d'une plus grande transparence. Financé par les primes, ce puissant lobby s'est rendu responsable de nombreux blocages qui perpétuent des situations devenues intolérables pour les assurés. rés en instaurant les règles claires que les atermoiements et demi-mesures politiques ont été incapables d'imposer. Elle mettra ainsi un terme aux incitations néfastes qui opposent fondamentalement les intérêts des assureurs-maladie à ceux de leurs assurés.

Le Parlement a déjà examiné trois propositions en

\section{«En séparant l'assurance complémentaire de l'assurance de base, il sera mis fin aux distorsions qui résultent de cette cohabitation.»}

\section{L'intérêt des assurés avant tout}

En séparant l'assurance complémentaire de l'assurance de base, il sera mis fin aux distorsions qui résultent de cette cohabitation et l'assurance de base sera rétablie dans sa véritable vocation mutualiste et sociale. Le débat politique retrouvera ainsi la sérénité indispensable à la poursuite des réformes. Mais, avant tout, cette séparation s'inscrit dans l'intérêt des assu- faveur d'une meilleure transparence. Elles ont successivement été retirées ou rejetées sans que les mesures prises ne permettent d'assurer la transparence de fonctionnement de l'assurance-maladie obligatoire. Il importe maintenant que le peuple fasse entendre sa voix, les lobbies parlementaires ayant fait échouer chacun de ces projets.
Nous vous invitons à signer et faire signer cette initiative «Pour la transparence de I'assurance-maladie»

Vous pouvez signer au moyen de la liste de signatures fournie avec le présent numéro ou en l'imprimant sur: http://initiative-transparence.ch

Les étiquettes autocollantes sont à porter visiblement pour faciliter l'entrée en matière avec vos interlocuteurs!
UNTERSCHREIBEN! initiative-transparenz.ch

SIGNEZ!

initiative-transparence.ch

FIRMARE!

iniziativa-trasparenza.ch 